\title{
Modality Specific Activations in Working Memory in Children with Cerebellar Medullobastoma: A Functional MRI Study
}

Duc Ha Hoang ${ }^{1,2}$, Anne Pagnier ${ }^{3}$, Emilie Cousin ${ }^{4,5}$, Karine Guichardet $^{3}$, Isabelle Schiff ${ }^{3}$, Fanny Dubois-Teklali ${ }^{3}$ and Alexandre Krainik ${ }^{1,2,4,6^{*}}$

${ }^{1}$ Grenoble Institute of Neurosciences, INSERM U836, Grenoble, France

${ }^{2}$ University Joseph Fourier, Grenoble, France

${ }^{3}$ Department of Pediatrics, Grenoble University Hospital, Grenoble, France

${ }^{4}$ UMS IRMaGe, Grenoble, France

${ }^{5}$ Laboratory of Psychology and Neurocognition, University Pierre Mendès, Grenoble, France

${ }^{6}$ Department of Neuroradiology and MRI, Grenoble University Hospital, Grenoble, France

\begin{abstract}
Introduction: Medulloblastomas are the most common malignant childhood brain tumors arising in the posterior fossa. As a result of advances in treatment the number of survivors has increased significantly. In this study, children treated for a cerebellar medulloblastoma demonstrated cognitive disorders in working memory, especially the visuospatial component, leading to impairments in school performance. This study aims to describe the cerebellar involvement in specific cognitive deficits observed in these children.
\end{abstract}

Method: Nine healthy volunteer children $(11.1 \pm 2.2$ years old $)$, were compared to 5 patients treated for cerebellar medulloblastoma (12.3 \pm 0.7 years old). All subjects were native French speakers, right-handed, with a global Intelligence Quotient (IQ) of 70-130. Stimuli were presented to the participants with alternating the sensory modality (visual, auditory) and the nature of communication (verbal, nonverbal) in a blocked 1-back design during functional Magnetic Resonance Imaging (fMRI) acquisitions. Results were analyzed using a Mann-Whitney U test for neuropsychological and behavioral data; SPM8 (Statistical Parametric Mapping) and SUIT (Spatially Unbiased Atlas Template) for anatomical and functional MRI data.

Results: Non-verbal working memory deficit was demonstrated in 4 of the 5 children treated for medulloblastoma. The 4 patients with working memory deficit all had surgery to the left posterior cerebellar lobe. The only patient without any working memory disorders did not have hemispheric cerebellar resection. Neuronal activations for nonverbal versus verbal contrast and visual versus auditory contrast were stronger in the left posterior cerebellar lobe. Brain activations in healthy subjects were consistent with the findings previously described in literature.

Conclusion: The cerebellum plays the same role in working memory in children as that has been previously described in adults. The left posterior cerebellar lobe may involve the visuospatial working memory. Based on neuroimaging and clinical studies, attention should be paid to avoid or limit the damage of the posterior cerebellar lobe to minimize cognitive deficits and improve the quality of life for children.

Keywords: Working memory; Children; Functional MRI; Cerebellum; Medulloblastoma

\section{Introduction}

Working Memory (WM) is a system of short-term information processing and storage involving the essential cognitive function of everyday life like thought, learning, and communication. WM involves three primary processes: encoding information, actively maintaining this information "on-line" in memory, and finally, using the information to guide behavior [1]. The model of WM most commonly used is the tripartite model of Baddeley and Hitch [2] which consists of three components: central administrator, phonological loop and visuo-spatial sketchpad (a fourth component: episodic buffer was later added to this model in 2000 [3]). In this study, the phonological loop and the visuo-spatial sketchpad were of particular interest and several tasks for assessing each domain in children have been established. The phonological loop or verbal WM has a role in language acquisition and has been shown to be important in learning new words and language [4] and the ability to hear and repeat an unfamiliar pseudoword in children [5]. The visuo-spatial sketchpad or nonverbal WM is responsible for maintaining the visual and spatial information as well as in the formation and manipulation of mental images [6].

Medulloblastomas, the most common primitive malignant tumors in children, are mainly located in cerebellum and classically treated by a combination of surgery, radiotherapy and/or chemotherapy [7]. Many factors affect the progressive intellectual reduction in survivors including: hydrocephalus, high radiation dose, large volume radiation and complementary chemotherapy, radiation vasculopathy and also young age at the time of treatment $[8,9]$. To improve quality-of-life outcomes, an assessment of WM in children undergoing treatment is important to prevent and limit cognitive impairment.

Functional MRI has become the main method to study brain function in healthy subjects and patients, especially in cognitive neuroscience. This method allows a better understanding of the neurophysiological bases of behavior in the pediatric population and has many advantages, most importantly being noninvasive. WM is considered as emerging from the interaction of the higher functions of sensory, attentional and memory components involving their specific brain regions. fMRI has been applied to better understand the role of

*Corresponding author: Alexandre Krainik, M.D, Ph.D, Department of Neuroradiology and MRI, Grenoble University Hospital, Chantourne Boulevard, BP 217, 38043 Grenoble, France, Tel: +33 4565205 86; Fax: +33 476765286 E-mail: akrainik@chu-grenoble.fr

Received December 07, 2013; Accepted January 23, 2014; Published January 27,2014

Citation: Hoang DH, Pagnier A, Cousin E, Guichardet K, Schiff I, et al. (2014) Modality Specific Activations in Working Memory in Children with Cerebella Medullobastoma: A Functional MRI Study. J Psychol Abnorm Child 3: 111. doi:10.4172/2329-9525.1000111

Copyright: @ 2014 Hoang DH, et al. This is an open-access article distributed under the terms of the Creative Commons Attribution License, which permits unrestricted use, distribution, and reproduction in any medium, provided the original author and source are credited. 
Citation: Hoang DH, Pagnier A, Cousin E, Guichardet K, Schiff I, et al. (2014) Modality Specific Activations in Working Memory in Children with Cerebellar Medullobastoma: A Functional MRI Study. J Psychol Abnorm Child 3: 111. doi:10.4172/2329-9525.1000111

Page 2 of 7

these brain regions in WM but has not been much used to study the functions of the cerebellum.

The cerebellum accounts only $10 \%$ the of total brain volume, but it contains about $80 \%$ of all neurons in the human brain [10] and it has an essential role in the central nervous system. The cerebellum has been traditionally considered to play a role in motor control, physical coordination and balance, but more recently it has been also considered to contribute to cognitive, emotional and language processing [11-13]. However, the role of the cerebellum in the visuo-spatial sketchpad has rarely been described. The number of medulloblastoma survivors has been increased by treatment advances and as a consequence they require more the rehabilitation. The objective of this study was to investigate the cerebellar contribution in WM disorders in children who had been treated for medulloblastoma by combining an anatomical and functional MRI study.

\section{Methods}

\section{Participants}

The neuropsychological and imaging data of healthy children volunteers ( 5 boys, 4 girls, mean of age $11.1 \mathrm{y}(\mathrm{SD}=2.2 \mathrm{y})$ ) was compared with patients with cerebellar medulloblastoma ( 5 boys, mean of age $12.3 y(S D=0.7 y))$ treated with surgery and radiotherapy $(5 / 5)$, +/- chemotherapy (3/5) in Grenoble, Lyon and St-Etienne pediatric oncology hospital units at least 6 months after the end of treatment. The handedness was determined by means of Delattolas laterality test [14]. The study has been approved by the local SUD EST V Ethic Committee and by the DGS (National Health Authorities). All of the children's parents/legal guardians provided written informed consent.

\section{Inclusion criteria}

The participants satisfied the conditions shown in Table 1.

\section{Neurologic assessment}

An onco pediatrician or neuro pediatrician at the participating centers carried out a clinical examination, including neurologic testing, on all patients and volunteers.

\section{Neuropsychological assessment}

The patients and controls were evaluated by a neuropsychological assessment protocol. It included an assessment of intellectual efficiency as well as an evaluation of memory and attentional functions by the tests that have been calibrated in a French population of the same age.

The overall assessment includes Wechsler Intelligence Scale for Children -WISC IV [15], laterality, Children's Memory Scale -CMS (history and location, immediate and delayed) [16], Grober and
Buschke procedure [17], A Alboy working memory test, Rey's figure [18] and TMT (Trail Making Test) [19]. These tasks (including resting phase) lasted about 4 hours per subject and were performed a maximum of two months before the fMRI.

\section{Imaging}

Task and stimuli: The participants were instructed to perform four classical n-back tasks (1-back tasks) using two modalities of presentation (visual vs. auditory) and two methods of displayed information (verbal vs. non-verbal). Four experimental tasks were tested: Auditory Verbal Task (AUVE), Auditory Non-Verbal (AUNV), Visual Verbal (VIVE) and Visual Non-Verbal (VINV). Each task was presented once during the experimental paradigm. The tasks included 28 stimuli, pseudorandomly presented. Visual tasks: each stimulus (word or spatial pattern) was centered on the middle of a black screen. Words were controlled in terms of frequency (high frequency) and were written in "Courier New" font, size 48 and spatial patterns were $2^{\circ}$ size. All visual stimuli were displayed on a computer monitor by using the E-prime software (E-prime Psychology Software Tools Inc., Pittsburgh, USA). The monitor was connected to a video-projector (Epson EMP 8200) permitting the transmission of visual stimuli into the magnet. The subject was able to visualize the stimuli by means of a projection screen situated behind the magnet and a mirror centered above the participant's eyes. Auditory conditions: (word or sound). The stimulus presentation time was 2000 $\mathrm{ms}$, with $3000 \mathrm{~ms}$ fixation point between items. All auditory stimuli were displayed by means of MR CONFON (http://www.mr-confon.de/ en/products.html) headphones. The participants were instructed to give manual instead of vocal responses, in order to avoid movement artifacts generated by articulation. The responses were generated by means of one key. Participants were required to respond when the current stimulus was identical to the previous. The manual responses were recorded, analyzed and the task performance evaluated according to the conditions (visual vs. auditory and verbal vs. non-verbal).

\section{MR acquisition}

The experiment was performed on a 1.5 T MR scanner (Philips) with 30 $\mathrm{mT} / \mathrm{m}$ gradient strength and a standard Philips head coil.

Functional image acquisition: fMRI scanning was performed with a gradient-echo/T2* weighted EPI method. Thirty-two adjacent axial slices parallel to the bi-commissural plane were acquired in interleaved mode. Slice thickness was $4 \mathrm{~mm}$. The voxel size was $4 \times 4 \times 4 \mathrm{~mm} ; 256$ $\mathrm{mm}$ field of view. The main sequence parameters were: $\mathrm{TR}=5 \mathrm{~s}, \mathrm{TE}=50$ ms, flip angle $=77^{\circ}, 56$ dynamics. Images were acquired during the 3 first seconds of each TR, 2 extant seconds for auditory and visual cues exposure. A "block" design was used during the fMRI session. Four runs were measured during the fMRI session corresponding to

\begin{tabular}{|l|l|l|}
\hline \multicolumn{1}{|c|}{ Conditions } & Healthy subject \\
\hline (i) native French speakers; & $\checkmark$ \\
\hline (ii) right-handed; & $\checkmark$ \\
\hline (iii) age of 8 to12 years 11 months; & $\checkmark$ \\
\hline (iv) the overall IQ from 70 to 130; & $\checkmark$ \\
\hline $\begin{array}{l}\text { (v) children treated for medulloblastoma after age of 6 years (homogeneous population in terms of brain maturation) and at least six } \\
\text { months after the end of all treatments; }\end{array}$ & $\checkmark$ \\
\hline (vi) having a good performance in the previous fMRI training; & \\
\hline $\begin{array}{l}\text { (vii) no contra-indications for MRI (for example: pacemaker, vascular clips, metal cardiac valve prosthesis, brain or cochlear stimulator, } \\
\text { dental prosthesis, other magnetizable prosthesis and claustrophobia rebel); }\end{array}$ & $\checkmark$ \\
\hline (viii) not taking methylphenidate (Ritalin $\circledast$ ) 48 hours before MRI; & $\checkmark$ \\
\hline (ix) without any history of psychiatric, neurological or other major medical disorder & $\checkmark$ \\
\hline
\end{tabular}

Table 1: inclusion criteria of participants for recent study. 
each task. Tasks were randomly presented across subjects. The total duration of each run was 4 min 40 seconds. During each run, the whole brain volume was measured 56 times and the field map obtained was subsequently used during data processing.

Anatomical image acquisition: Finally, a T1-weighted highresolution three-dimensional anatomical volume was acquired using a $3 \mathrm{D}$ gradient echo inversion recovery sequence (field of view $=256 \mathrm{~mm}$; resolution: $1 \times 1 \times 1 \mathrm{~mm}$; 128 axial spiral slices).

\section{Data analysis}

Spatial pre-processing: Data analysis was performed using the general linear model as implemented in SPM8 (Welcome Department of Imaging Neuroscience, London, UK, www.fil.ion.ucl.ac.uk/spm) where each event is modeled using a hemodynamic function model. Data analysis started with several spatial pre-processing steps. All volumes were realigned to correct head motion using rigid body transformations. After discarding the four first slices for the scanner to reach equilibrium, the first volume of the first fMRI session was taken as a reference volume (i.e. this volume was originally the fifth volume). Weighted anatomical volume was co-registered to mean images created by the realignment procedure and was normalized to the MNI space using a trilinear interpolation. The anatomical normalization parameters were subsequently used for the normalization of functional volumes. Finally, each functional volume was smoothed by an $8-\mathrm{mm}$ FWHM (Full Width at Half Maximum) Gaussian kernel to ameliorate differences in inter subject localization. The time series for each voxel were high-pass filtered $(1 / 128 \mathrm{~Hz}$ cutoff) to remove low-frequency noise and signal drift.

Statistical analysis: For each fMRI session, two experimental conditions were defined: Rest (R) and n-back Task (T). These conditions were modeled as two regressors and convolved with the canonical form of the Hemodynamic Response Function (HRF).

A statistical analysis was performed at an individual level for each fMRI session corresponding to each task (VIVE, VINV, AUVE and AUNV). The general linear model was used to generate the parameter estimates of activity at each voxel, for each condition, and each participant (healthy and pathological participants). Moreover, as additional factors, movement parameters derived from the realignment corrections ( 3 translations and 3 rotations) were also taken into account in the design matrix. Subsequently, [T-R] contrasts were calculated for each task and participant. The modality of presentation (visual, auditory) and the nature of information (verbal, non-verbal) in the four experimental conditions: VIVE, VINV, AUVE and AUNV were defined and a flexible design analysis on the contrast images derived from individual analyses in healthy participants was performed. Subsequently, two types of main effects were calculated:

Main effect of modality of presentation: The contrast [AU $(\mathrm{VE}+\mathrm{NV})$ vs. VI $(\mathrm{VE}+\mathrm{NV})]$ was calculated in order to identify modality specific activations in healthy children. The opposite contrast [VI $(\mathrm{Ve}+\mathrm{NV})$ vs. $\mathrm{AU}(\mathrm{Ve}+\mathrm{NV})]$ was also calculated.

Main effect of nature of information: The contrast [VE (AU+VI) vs. $\mathrm{NV}(\mathrm{AU}+\mathrm{VI})$ ] was calculated in order to explore the cerebral regions required in $\mathrm{WM}$ processing according to the nature of information and similar calculation for the opposite contrast [NV (AU+VI) vs. VE $(\mathrm{AU}+\mathrm{VI})]$.

The anatomical location of the activated regions revealed by the main effects was determined by using the xj view toolbox (http:// www.alivelearn.net/xjview8/) allowing visualization of anatomical,
Brodmann areas and MNI coordinates of the activations. In addition, the Spatially Unbiased Atlas Template (SUIT) [20] was used to view cerebellar topography with BOLD activations.

Anatomical data analysis: The Spatially Unbiased Atlas Template (SUIT) of the cerebellum and brainstem [20] for the SPM segmentation method and the MRI Atlas of the Human Cerebellum [21]were used to localize individual cerebellar lesions and to overlap these lesions onto the normal cerebellar template obtained in the control group. The same method was used for localizing the activation patterns from the SPM method within the cerebellum which were visualized using MRIcroGL software (http://www.cabiatl.com/mricrogl/).

The variables did not have a Gaussian distribution and they were not all parametric, therefore, the Mann-Whitney $U$ test was chosen for analyzing neuropsychological and behavioral data.

\section{Results}

9 patients and 12 healthy controls were entered into the study. Of these 4 patients and 3 healthy controls were excluded because of: excessive movement $(n=3)$, left-handedness $(n=1)$, anxiety $(n=2)$, lack of task performance $(n=1)$, leaving 9 healthy children volunteers $(5$ boys, 4 girls, mean of age $11.1 \mathrm{y}(\mathrm{SD}=2.2 \mathrm{y})$ and 5 patients ( 5 boys, mean of age $12.1 \mathrm{y}(\mathrm{SD}=0.6 \mathrm{y})$ suitable for analysis.

\section{Neuropsychological results}

Weschler intelligence scale (IQ) : In the control and patient group, the Weschler (VCI and PRI) neuropsychological tests showed normal scores for age and no difference between two groups in the perceptual reasoning index (PRI) $(\mathrm{U}=16.5, \mathrm{Z}=1.41 \mathrm{p}=0.16$, Mann-Whitney $\mathrm{U}$ test). However, in the Verbal Comprehension Index (VCI), the patient group performance score $(93.512 .03)$ was significantly lower than the control group $(110.4 \pm 8.73)(\mathrm{U}=5, \mathrm{Z}=2.65, \mathrm{p}=0.007$, Mann-Whitney $\mathrm{U}$ test $)$.

Working memory test: In the tests related to the phonological loop, there was no significant difference in performance score between the control group and the patient group. However, in the Compound Stimulus Visual Information task (CSVI) involving the visuospatial sketchpad, there was a significant difference in pattern test with the patient group performance significantly lower than the control group ( $\mathrm{U}=11, \mathrm{Z}=2, \mathrm{p}=0.04$, Mann-Whitney $\mathrm{U}$ test).

Behavioral results: The accuracy and Reaction Time (RT) were calculated for each participant and for the 4 tasks (VIVE, VINV, AUVE and AUNV). All subjects responded with high accuracy for each task (more than $90 \%$ ). The Mann-Whitney $U$ test showed a significant difference for two condition VINV $(\mathrm{U}=3343, \mathrm{Z}=-2, \mathrm{p}=0.04)$ and $\operatorname{AUNV}(\mathrm{U}=3327, \mathrm{Z}=-2, \mathrm{p}=0.04)$ which means that the reaction time to perform the nonverbal task in the patient group was significantly longer than in the control group.

Anatomical and functional MRI results in the patient group: The cerebellar lesion topography for 5 patients is shown in Figure 1. In the patient group, all 4 patients with principal resected lesions in left inferior cerebellar lobe (lobule VIIB/VIII and Crus I/II) had WM impairment. The only patient with no WM deficit had an inferior vermis resection and no surgery in the left inferior cerebellar lobe. For each task and contrast, the activations maps showed a reduction of cluster size in the patients group vs. healthy children group (Figure 1).

Functional MRI results in the healthy control group: A BOLD signal in the cerebellum and brain for each task (VIVE, VINV, AUVE and AUNV) was obtained for four contrasts (visual vs. auditory, auditory vs. visual, verbal vs. nonverbal and nonverbal vs. verbal) from data processing by SPM8. 
Citation: Hoang DH, Pagnier A, Cousin E, Guichardet K, Schiff I, et al. (2014) Modality Specific Activations in Working Memory in Children with Cerebellar Medullobastoma: A Functional MRI Study. J Psychol Abnorm Child 3: 111. doi:10.4172/2329-9525.1000111

Page 4 of 7

BOLD activation patterns within the cerebellum: Analyzing the contrast for both the effects of presentation modality and nature of information, it was found that the visual vs. auditory contrast exhibited greater activations in the left posterior cerebellar lobe (lobules VIIb, VIII, Crus I) and other regions. In the nonverbal vs. verbal contrast, robust activations in lobule VI, VIIb, Crus I/II occurred (Figure 2).

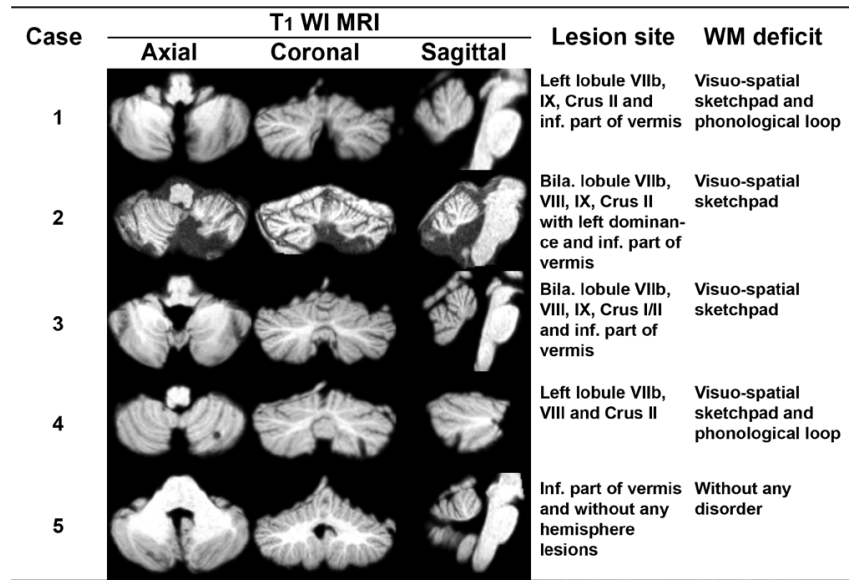

Figure 1: MRI images. Anatomical lesion mapping of patient group with lobules labelled from the Spatially Unbiased Infratentorial Template (SUIT) of the cerebellum and brainstem (method of Diedrichsen et al. [22]) and being visualized on MRICroN software.

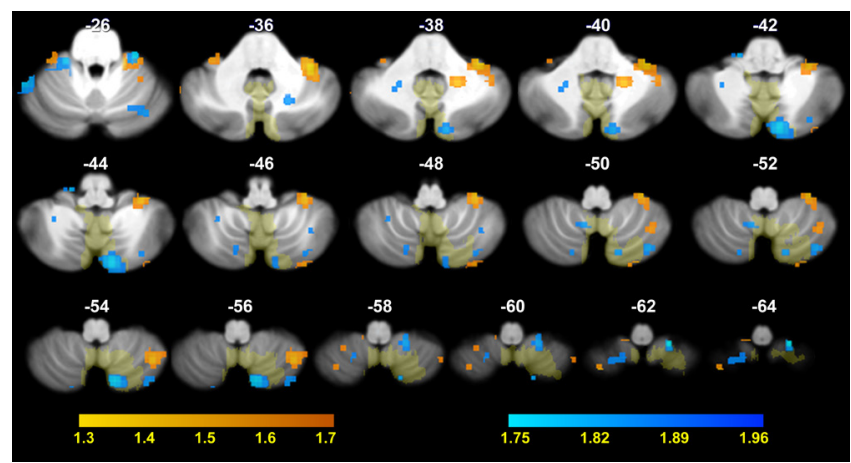

Figure 2: fMRI results. Overlaps on MRICRoN of resected lesion area (yellow) activations observed in nonverbal vs. verbal tasks contrast (blue clusters) and in visual vs. auditory contrast (orange clusters). Activation maps are threshold at a voxel-level threshold of $\mathrm{P}<0.05$ (uncorrected) with a cluster-size $>4$.

\section{BOLD activation patterns within the brain}

Visual verbal tasks (VIVE) and Visual non-verbal tasks (VINV): BOLD activations were concentrated mainly in the parietal-frontal region during the VIVE tasks and the VINV tasks. During the two tasks, stronger activations were found bilaterally in the Inferior Frontal Gyrus (IFG) (BA45/47), Middle Frontal Gyrus (MFG) (BA46), Inferior Parietal Lobule (IPL) (BA40) and the fusiform gyrus (BA17/18).

Auditory verbal tasks (AUVE) and Auditory non-verbal tasks (AUNV): These two presentations activated some robust clusters in the bilateral temporo-frontal regions, particular in the Superior Temporal Gyrus (STG) (BA 21/22), IFG (BA 47) and IPL (BA 40). These activations predominated on the left side for AUVE tasks and on the right side for AUNV tasks. They were greater during AUNV tasks than during AUVE tasks.

Main effect of modality of presentation(visual /auditory): Compared to auditory tasks, visual tasks produced greater BOLD signals in the bilateral parieto-occipital areas (BA17/18/19), especially the bilateral primary visual cortex, bilateral superior and inferior frontal gyrus, bilateral fusiform gyrus and left MFG (Table 2). On the other hand, auditory vs. visual contrast produced prominent activations in bilateral parieto-temporal areas, especially the bilateral primary auditory cortex (BA41/42), Wernicke's area (BA22) as well as bilateral STG $(\mathrm{BA} 21 / 22)$

Main effect of nature of information (verbal/ non-verbal): Compared to nonverbal tasks, common activations by verbal tasks were bilaterally observed in the MTG (BA 21/22), supramarginal gyrus (BA 40) with left-sided predominance whereas nonverbal vs. verbal contrast activated predominately the right middle occipital gyrus (BA18/19), right fusiform gyrus and right lingual gyrus (BA17).

\section{Discussion}

The cerebellum was assumed to have an important role in WM disorders in children undergoing surgery for cerebellar medulloblastoma. In this study, children who had surgery for their cerebellar lesions had WM impairment, especially in the visuo-spatial memory or nonverbal WM. This anatomical and functional imaging study was performed to better understand the cerebellar involvement in working memory disorders in children treated for medulloblastoma.

\section{The cerebellum plays an important role in working memory}

The cognitive function of the cerebellum is now much better

\begin{tabular}{|c|c|c|}
\hline Task domain & Location within cerebellum & Reported by \\
\hline \multirow{4}{*}{$\begin{array}{l}\text { Nonverbal WM } \\
\text { (visuo-spatial sketchpad) }\end{array}$} & Bilateral lobule VI, VIIb, VIII, IX, Crus I and II & Hautzel et al. [39] \\
\hline & Left cerebellum & Ribaupierre et al. [40] \\
\hline & Cerebellum & Levisohn et al. [31] \\
\hline & Posterior cerebellum & Schmahmann and Sherman [32] \\
\hline \multirow{5}{*}{$\begin{array}{l}\text { Verbal WM } \\
\text { (Phonological loop) }\end{array}$} & Bilateral lobule VI, VIIb, VIII, Crus I and II & Hautzel et al. [39] \\
\hline & Bilateral lobule VI, VII, VIII and right IX & Cooper et al. [41] \\
\hline & Left lobule VIII & Kirschen et al. [27] \\
\hline & Left lobule VIII & Kirschen et al. [26] \\
\hline & Right lobule VI and Crus I & Thürling et al. [30] \\
\hline \multirow{2}{*}{ Language processing } & Right posterolateral cerebellum & Stoodley and Schmahmann $[37,40]$ \\
\hline & Right lobule VI, Crus I and II & Stoodley et al. [38] \\
\hline \multirow{2}{*}{ Spatial processing } & Left posterolateral cerebellum & Stoodley and Schmahmann [37] \\
\hline & Left lobule VI and VII & Stoodley et al.[38] \\
\hline
\end{tabular}

Table 2: Examples of tasks during which cerebellar activation is reported in literature and the location of the activation patterns within the cerebellum. 
understood and several recent studies have found evidence of the cerebellar role in cognitive function [23-27], including auditory processing [28], phonological loop [27,29,30] and visuospatial sketchpad [31]. However, the specific contributions of different cerebellar regions to the different phases of WM have not been clearly defined and it may differ depending on the demands of the WM tasks [30].

In this study, using neuropsychological tests and behavior results, the four patients with the main lesions located in the left posterior cerebellar hemisphere lobe (lobule VIIb, VIII, IX and Crus I/II) and inferior part of vermis had significant impairment of visuo-spatial WM compared to healthy subjects. Furthermore, the only patient without any lesion of the posterior cerebellar hemisphere did not have any WM deficit, even though this patient had received radiotherapy and chemotherapy doses like others patients. In the healthy subject group, greater neuronal activations were shown in the left posterior cerebellar hemisphere for visual vs. auditory contrast and nonverbal vs. verbal contrast. This suggests that left posterior cerebellar damage may induce visuo-spatial problems. These finding were comparable with other results in the literature. For example, Schmahmann and Sherman [32] found that cerebellar cognitive affective syndrome with visuospatial disorganization and impaired visuospatial memory was seen in patients with posterior cerebellar lobe lesion. Hokkanen et al. [33] found that patients with left cerebellar lesion performed slowly in a visuospatial task and Wallesch and Horn [34] reported that an excision of the left cerebellar hemisphere tumors evoked visual spatial deficits. On other hand, this study demonstrated the left-right lateralization of cerebellum in verbal and nonverbal WM. The verbal WM activated predominantly the right posterior cerebellar lobe while the nonverbal WM activated the opposite side of the cerebellum [35-38]. This is summarized in Table 2 .

The results of this study differ from some published results because of the different methods used in evaluating the patient's WM. For example, Kirschen et al. [26] stated that damage to left hemispheral lobule VIII was associated with reduced digit span to auditory stimuli and phonological storage (phonological loop component), but they used verbal tasks (including only a series of words) to evaluate the verbal WM in patients with cerebellar damage. Ravizza et al. [42] showed that lesions of the right cerebellar hemisphere would be more disruptive to verbal WM than lesions of the left cerebellar hemisphere (but in elderly patients). On the other hand, Hautzel et al. [39] provided evidence of cognitive involvement of the cerebellum in WM but didn't find the cerebellar lateralization for different WM components.

\section{BOLD activations in brain}

Modality of visual and auditory presentation: The results of this study provide strong evidence that visual tasks activated bilaterally the occipital cortex, IFG (BA45/47), IPL (BA40) and fusiform gyrus. These outcomes demonstrate the role of the occipital cortex in visual ability and were also found in previous studies $[43,27]$. Common clusters in the lingual gyrus, fusiform gyrus, superior and IPL were found with a slight predominance on the right side; the bilateral cuneus, precuneus and MFG with a slight predominance on the right side. These findings were similar to those of other studies [44,45].

During the auditory presentation, bilateral STG, the region classically known as the primary auditory area was activated and that is consistent with other studies $[46,47]$. Furthermore, the STG is involved in many critical functions in hearing, speech, language and in the integration of auditory and visual cues [48]. Thus, the visual $\mathrm{n}$-back tasks elicited greater activation in occipital regions, whereas the auditory tasks did the same in the STG. This was confirmed by
Rodriguez-Jimenez et al. [49]. The auditory stimuli also activated the IFG which plays an important role in cognitive function [50], semantic WM [51], language processing [52], auditory processing [46], both visual and auditory WM [27].

Overall, the fronto-parietal cortex, especially the IPL (BA40) and the IFG (BA45/47), plays an important role in WM, in which auditory WM predominates in the STG and visual WM mainly activates the occipital cortex.Nature of verbal and nonverbal information: The verbal presentation predominated on the left side hemisphere whereas the nonverbal presentation predominated on the right side of brain. This result demonstrates a left-right lateralization in the verbal and nonverbal WM, which agrees well with results of other studies [46,47,53-55]. The activations during nonverbal stimuli were greater than those during verbal stimuli reflecting possibly an increased difficulty in identifying nonverbal compared to verbal tasks. This was consistent with behaviour results which revealed slower reaction times and lower accuracies for the non-verbal conditions vs. the verbal conditions.

Both verbal and nonverbal tasks activated the middle temporal gyrus (BA 21/22) suggesting a role for this region in language reception and processing. This region is involved in verbal and non-verbal semantic cognition [56]. The activations were found bilaterally in the Lateral Prefrontal Cortex (LPFC) (including the BA44 and BA40) during two tasks with predominance in verbal stimuli presentation, suggesting an important role of the DLPFC in verbal WM [53,57-59]. D'Esposito et al. [58] showed in more detail that the dorsal prefrontal cortex was involved in spatial WM and the ventral prefrontal cortex involved in the verbal WM. In an animal study, Goldman-Rakic [60] found the lateral prefrontal cortex and the middle frontal gyrus (BA 46) to be responsible for the phonological loop and the ventral inferior frontal gyrus (BA 44/45/47) to be in charge of the visuospatial sketchpad. The visuo-spatial sketchpad is activated in the right inferior parietal lobule [61] and bilateral posterior parietal regions with righthemispheric dominance [62]. Taken together, these finding revealed that each task evoked some specific zones in the cerebellum and the brain reinforcing the existence of the cerebro-cerebellar circuit in WM function as described in the literature $[27,25,63]$.

In this study, the effects of cerebellar irradiation were not evaluated but in the literature, some studies on irradiation and chemotherapy effects in patients with brain lesion (acute lymphoblastic leukemia [64], cerebellar tumors [31], medulloblastoma [65]) have demonstrated a correlation between these effects and WM deficit. A linear reduction of signal BOLD in the primary visual cortex was also found in the patient group compared to the healthy children group [66]. Thus, patients with posterior fossa malignant tumors can acquire damage in the central nervous system from the tumor's growth, per-surgical resection of the tumors, the effects of chemotherapy and radiotherapy, or from a combination of any of these factors [67]. WM disorders in medulloblastoma survivors can result from a combination of many factors but in this study, we demonstrated the importance of cerebellar damage after surgery. Due to ethical constraints, the assessment of WM disorders used by each specific factor difficult to perform. There habilitation for these patients there for also needs to take account of all possible risk factors.

In summary, the present study provides further evidence that the cerebellum plays a role in WM in children similar to that already described for adults. Damage to the left posterior cerebellar lobe may impair nonverbal WM performance and children with cerebellar lesions should routinely undergo long-term monitoring of their intellectual development. Secondly, these results reinforce the existence of functional cerebro-cerebellar networks during WM tasks. 
Citation: Hoang DH, Pagnier A, Cousin E, Guichardet K, Schiff I, et al. (2014) Modality Specific Activations in Working Memory in Children with Cerebellar Medullobastoma: A Functional MRI Study. J Psychol Abnorm Child 3: 111. doi:10.4172/2329-9525.1000111

\section{Limitations}

Limitations of the present study included: (i) low statistical power of the results because of the difficulty of inclusion (rare diseases), difficulties in performing neuropsychological tests and fMRI acquisitions (multiple exclusion criteria) and limited power of MRI machine $(1.5 \mathrm{~T})$; (ii) it was difficult to distinguish the surgery effects from those of radiotherapy because of the absence of a "surgery alone group"; (iii) 1-back tasks, although easier perform, (low WM load) may not be the most effective to evaluate WM performance.

\section{Acknowledgement}

This work was supported by grants from French Society for Childhood Cancer (SFCE) and the Hospital Clinical Research Program (PHRC) of France.

\section{References}

1. van den Bosch GE, Marroun HE, Schmidt MN, Tibboel D, Manoach DS, et al. (2014) Brain connectivity during verbal working memory in children and adolescents. Hum Brain Mapp 35: 698-711.

2. McGettigan C, Warren JE, Eisner F, Marshall CR, Shanmugalingam $P$, et al (2011) Neural correlates of sublexical processing in phonological working memory. J Cogn Neurosci 23: 961-977.

3. Baddeley A (2000) The episodic buffer: a new component of working memory? Trends Cogn Sci 4: 417-423.

4. Service $E$ (1992) Phonology, working memory, and foreign-language learning. Q J Exp Psychol A 45: 21-50

5. Gathercole SE, Adams AM, Hitch GJ (1994) Do young children rehearse? An individual-differences analysis. Mem Cognit 22: 201-207.

6. Roulin JL, Monnier C (1994) Ã la recherche du calepinvisuo-spatial en memoire de travail. Annee Psychol 94:425-460.

7. Massimino M, Giangaspero F, Garrè ML, Gandola L, Poggi G, et al. (2011) Childhood medulloblastoma. Crit Rev Oncol Hematol 79: 65-83.

8. Duffner PK (2010) Risk factors for cognitive decline in children treated for brain tumors. Eur J Paediatr Neurol 14: 106-115.

9. George AP, Kuehn SM, Vassilyadi M, Richards PM, Parlow SE, et al. (2003) Cognitive sequelae in children with posterior fossa tumors. Pediatr Neurol 28: 42-47.

10. Azevedo FA, Carvalho LR, Grinberg LT, Farfel JM, Ferretti RE, et al. (2009) Equal numbers of neuronal and nonneuronal cells make the human brain an isometrically scaled-up primate brain. J Comp Neurol 513: 532-541.

11. Schmahmann JD, Caplan D (2006) Cognition, emotion and the cerebellum Brain 129: 290-292.

12. Rapoport M, van Reekum R, Mayberg H (2000) The role of the cerebellum in cognition and behavior: a selective review. J Neuropsychiatry Clin Neurosci 12: 193-198

13. Stoodley CJ (2012) The cerebellum and cognition: evidence from functional imaging studies. Cerebellum 11: 352-365.

14. De Agostini Maria, Dellatolas Georges (1988) Uneepreuve simple pour evaluer la preference manuelle chez l'enfant Ã partir de 3 ans. Enfance 41:139-147.

15. Wechsler D (2005) Echelled'intelligence de Wechsler pour enfants WISC-IV. (4th edn), Paris: ECPA - Editions du Centre de PsychologieAppliquee.

16. http://link.springer.com/referenceworkentry/10.1007/978-0-387-79948-3_1532

17. Grober E, Buschke H (1987) Genuine memory deficits in dementia. Dev Neuropsychol 3:13-36.

18. Rey A (1941) The psychological examination in cases of traumatic encepholopathy. Problems. Arch Psychol 28: 215-285.

19. Reitan RM (1971) Trail making test results for normal and brain-damaged children. Percept Mot Skills 33: 575-581.

20. Diedrichsen J, Balsters JH, Flavell J, Cussans E, Ramnani N (2009) A probabilistic MR atlas of the human cerebellum. Neuroimage 46: 39-46.

21. Schmahmann JD, Doyon J, McDonald D, Holmes C, Lavoie K, et al. (1999) Three-dimensional MRI atlas of the human cerebellum in proportional stereotaxic space. Neuroimage 10: 233-260.
22. Diedrichsen $J$ (2006) A spatially unbiased atlas template of the human cerebellum. Neuro Image 33:127-138.

23. Schmahmann JD, Weilburg JB, Sherman JC (2007) The neuropsychiatry of the cerebellum - insights from the clinic. Cerebellum 6: 254-267.

24. Schmahmann JD (2010) The role of the cerebellum in cognition and emotion personal reflections since 1982 on the dysmetria of thought hypothesis, and its historical evolution from theory to therapy. Neuropsychol Rev 20: 236-260.

25. O'Reilly JX, Beckmann CF, Tomassini V, Ramnani N, Johansen-Berg H (2010) Distinct and overlapping functional zones in the cerebellum defined by resting state functional connectivity. Cereb Cortex 20: 953-965.

26. Kirschen MP, Davis-Ratner MS, Milner MW, Chen SH, Schraedley-Desmond $\mathrm{P}$, et al. (2008) Verbal memory impairments in children after cerebellar tumor resection. Behav Neurol 20: 39-53.

27. Kirschen MP, Chen SH, Desmond JE (2010) Modality specific cerebrocerebellar activations in verbal working memory: an fMRI study. Behav Neurol 23: $51-63$

28. Petacchi A, Laird AR, Fox PT, Bower JM (2005) Cerebellum and auditory function: an ALE meta-analysis of functional neuroimaging studies. Hum Brain Mapp 25: 118-128.

29. Marvel CL, Desmond JE (2010) Functional topography of the cerebellum in verbal working memory. Neuropsychol Rev 20: 271-279.

30. Thürling M, Hautzel H, Küper M, Stefanescu MR, Maderwald S, et al. (2012 Involvement of the cerebellar cortex and nuclei in verbal and visuospatial working memory: a 7 T fMRI study. Neuroimage 62: 1537-1550.

31. Levisohn L, Cronin-Golomb A, Schmahmann JD (2000) Neuropsychological consequences of cerebellar tumour resection in children: cerebellar cognitive affective syndrome in a paediatric population. Brain J Neurol 123: 1041-1050.

32. Schmahmann JD, Sherman JC (1998) The cerebellar cognitive affective syndrome. Brain J Neurol 121: 561-579.

33. Hokkanen LS, Kauranen V, Roine RO, Salonen O, Kotila M (2006) Subtle cognitive deficits after cerebellar infarcts. Eur J Neurol 13: 161-170.

34. Wallesch CW, Horn A (1990) Long-term effects of cerebellar pathology on cognitive functions. Brain Cogn 14: 19-25.

35. Riva D, Giorgi C (2000) The cerebellum contributes to higher functions during development: evidence from a series of children surgically treated for posterior fossa tumours. Brain 123: 1051-1061.

36. Scott RB, Stoodley CJ, Anslow P, Paul C, Stein JF, et al. (2001) Lateralized cognitive deficits in children following cerebellar lesions. Dev Med Child Neurol 43: 685-691.

37. Stoodley CJ, Schmahmann JD (2009) Functional topography in the human cerebellum: a meta-analysis of neuroimaging studies. Neuroimage 44: 489501.

38. Stoodley CJ, Valera EM, Schmahmann JD (2012) Functional topography of the cerebellum for motor and cognitive tasks: an fMRI study. Neuroimage 59 : $1560-1570$.

39. Hautzel H, Mottaghy FM, Specht K, Müller HW, Krause BJ (2009) Evidence of a modality-dependent role of the cerebellum in working memory? An fMR study comparing verbal and abstract n-back tasks. Neuroimage 47: 2073-2082.

40. deRibaupierre S, Ryser C, Villemure JG, Clarke S (2008) Cerebellar lesions: is there a lateralisation effect on memory deficits? Acta Neurochir (Wien) 150: $545-550$.

41. Cooper FE, Grube M, Von Kriegstein K, Kumar S, English P, et al. (2012 Distinct critical cerebellar subregions for components of verbal working memory. Neuropsychologia 50: 189-197.

42. Ravizza SM, McCormick CA, Schlerf JE, Justus T, Ivry RB, et al. (2006) Cerebellar damage produces selective deficits in verbal working memory. Brain 129: 306-320.

43. Burton MW, Locasto PC, Krebs-Noble D, Gullapalli RP (2005) A systematic investigation of the functional neuroanatomy of auditory and visual phonological processing. Neuroimage 26: 647-661.

44. Ganis G, Thompson WL, Kosslyn SM (2004) Brain areas underlying visua mental imagery and visual perception: an fMRI study. Brain Res Cogn Brain Res 20: 226-241.

45. Barban F, Daniele Zannino G, Macaluso E, Caltagirone C, Carlesimo GA 
Citation: Hoang DH, Pagnier A, Cousin E, Guichardet K, Schiff I, et al. (2014) Modality Specific Activations in Working Memory in Children with Cerebellar Medullobastoma: A Functional MRI Study. J Psychol Abnorm Child 3: 111. doi:10.4172/2329-9525.1000111

(2013) Letters persistence after physical offset: visual word form area and left planumtemporale. An fMRI study. Hum Brain Mapp 34: 1282-1292.

46. Fakhri M, Sikaroodi H, Maleki F, Ghanaati H, Oghabian MA (2013) Impacts of normal aging on different working memory tasks: implications from an fMRI study. Behav Neurol 27: 235-244.

47. Rogalsky C, Rong F, Saberi K, Hickok G (2011) Functional anatomy of language and music perception: temporal and structural factors investigated using functional magnetic resonance imaging. J Neurosci 31: 3843-3852.

48. Robins DL, Hunyadi E, Schultz RT (2009) Superior temporal activation in response to dynamic audio-visual emotional cues. Brain Cogn 69: 269-278.

49. Rodriguez-Jimenez R, Avila C, Garcia-Navarro C, Bagney A, Aragon AM, et al. (2009) Differential dorsolateral prefrontal cortex activation during a verbal n-back task according to sensory modality. Behav Brain Res 205: 299-302.

50. Derrfuss J, Brass M, von Cramon DY (2004) Cognitive control in the posterior frontolateral cortex: evidence from common activations in task coordination, interference control, and working memory. Neuroimage 23: 604-612.

51. Fiebach CJ, Friederici AD, Smith EE, Swinney D (2007) Lateral inferotemporal cortex maintains conceptual-semantic representations in verbal working memory. J Cogn Neurosci 19: 2035-2049.

52. Petrides M, Pandya DN (2002) Comparative cytoarchitectonic analysis of the human and the macaque ventrolateral prefrontal cortex and corticocortical connection patterns in the monkey. Eur J Neurosci 16: 291-310.

53. Rothmayr C, Baumann O, Endestad T, Rutschmann RM, Magnussen S, et al. (2007) Dissociation of neural correlates of verbal and non-verbal visual working memory with different delays. Behav Brain Funct 3: 56.

54. Floel A, Poeppel D, Buffalo EA, Braun A, Wu CW, et al. (2004) Prefrontal cortex asymmetry for memory encoding of words and abstract shapes. Cereb Cortex 14: 404-409.

55. Habeck C, Rakitin B, Steffener J, Stern Y (2012) Contrasting visual working memory for verbal and non-verbal material with multivariate analysis of fMRI. Brain Res 1467: 27-41.
56. Hoffman P, Pobric G, Drakesmith M, Lambon Ralph MA (2012) Posterio middle temporal gyrus is involved in verbal and non-verbal semantic cognition: Evidence from rTMS. Aphasiology 26: 1119-1130.

57. Baddeley A (2003) Working memory: looking back and looking forward. Nat Rev Neurosci 4: 829-839.

58. D'Esposito M, Aguirre GK, Zarahn E, Ballard D, Shin RK, et al. (1998) Functional MRI studies of spatial and nonspatial working memory. Brain Res Cogn Brain Res 7: 1-13.

59. McNab F, Klingberg T (2008) Prefrontal cortex and basal ganglia control access to working memory. Nat Neurosci 11: 103-107.

60. Goldman-Rakic PS (2011) Circuitry of Primate Prefrontal Cortex and Regulation of Behavior by Representational Memory. John Wiley \& Sons

61. Smith EE, Jonides J (1998) Neuroimaging analyses of human working memory. ProcNatlAcadSci U S A 95: 12061-12068.

62. Ebner K, Lidzba K, Hauser TK, Wilke M (2011) Assessing language and visuospatial functions with one task: a "dual use" approach to performing fMRI in children. Neuroimage 58: 923-929.

63. Chen SH, Desmond JE (2005) Cerebrocerebellar networks during articulatory rehearsal and verbal working memory tasks. Neuroimage 24: 332-338.

64. Ashford J, Schoffstall C, Reddick WE, Leone C, Laningham FH, et al. (2010) Attention and working memory abilities in children treated for acute lymphoblastic leukemia. Cancer 116: 4638-4645.

65. Riva D, Giorgi C, Nichelli F, Bulgheroni S, Massimino M, et al. (2002) Intrathecal methotrexate affects cognitive function in children with medulloblastoma. Neurology 59: 48-53.

66. Zou P, Mulhern RK, Butler RW, Li CS, Langston JW, et al. (2005) BOLD responses to visual stimulation in survivors of childhood cancer. Neuroimage 24: 61-69.

67. Cantelmi D, Schweizer TA, Cusimano MD (2008) Role of the cerebellum in the neurocognitive sequelae of treatment of tumours of the posterior fossa: an update. Lancet Oncol 9: 569-576. 